\title{
EVALUATION OF BASAL DENTAL IMPLANT PLACEMENT IN BASAL BONE OF ATROPHIC ALVEOLAR RIDGE
}

\author{
Salah Abdallah Saad Yousif ${ }^{1^{*}}$ Wael Ahmed Elmohandes ${ }^{2}$, Ahmed Mohammed Hosny ${ }^{3}$
}

\begin{abstract}
Objectives: This study was designed for radiographic and clinical evaluation of basal dental implant placement in atrophic alveolar ridge. Subjects and methods: The present study was conducted on twenty implants for individuals presented with sever bony defect and seeking for dental implant. Preoperative clinical evaluation was carried out; both medical and dental histories were taken and investigated. Evaluation of the interocclusal space for the placement of the prosthesis. Panoramic radiograph and CBCT scan for ridge evaluation and planning, Measuring height and width of residual alveolar ridge was performed in CBCT. Surgical stent were fabricated with aid of $\mathrm{CBCT}$ to act as a guide for simple and accurate orientation and angulation during implant placement and to preserve vital structures. The surgical guide was seated in the patient mouth at its position, the implant site was drilled through the holes designed in the surgical stent and implant then positioned. After surgery, initial stability was estimated by periotest, immediate postsurgical panoramic and CBCT radiograph to evaluate position of the implant. Follow up after 6 months was done to denote stability and marginal bone loss. Results: Periotest readings refer to excellent stability immediately after implant and also at six months later. Conclusion: Good stability in basal implants is related to its design permitting cortical bone engagement. Furthermore, it is a compression screw thus it corticalizes the spongious bone around the implant. Basal implant can be used successfully in severely atrophied ridge and compromised bone. Basal implants are used to avoid any extra surgical procedures, because these implants anchor reliably in resorption stable cortical bone and they are loaded immediatly after surgery.
\end{abstract}

KEY WORDS: GBR, augmentation of alveolar bone , basal implant, BCS implant.

\section{INTRODUCTION}

When teeth are lost or extracted alveolar atrophy occurs ${ }^{(1,2)}$. The reduction of the alveolar bone occurs at the buccolingual as well as apicocoronal dimension of the alveolar ridge at the edentulous site ultimately resulting in a short and narrow alveolar ridge ${ }^{(3,4)}$ as it well known the amount of bone surrounding the implant and the quality of that bone ${ }^{(5)}$.
The size $^{(6)}$, and type ${ }^{(7)}$ of implant seriously influence the stability of an implant, and whether it is associated with one or two bony cortices ${ }^{(8)}$. Implant length has proved to be an important factor for the success of implantation, particularly for the atrophied posterior maxillary area ${ }^{(9)}$.

In situations with alveolar bone atrophy, there is reduction in bone height and width thus, there is no adequate bone for supporting the implant.

1. Masters Candidate, Dept. of Oral and Maxillofacial Surgery, Faculty of Dental Medicine, Cairo, boys, Al-Azhar University.

2. Professor of Oral and Maxillofacial Surgery, Faculty of Dental Medicine, Cairo, boys, Al-Azhar University.

3. Lecturer of Oral and Maxillofacial Surgery, Faculty of Dental Medicine, Cairo, boys, Al-Azhar University.

-Corresponding author: dr.salah2100@gmail.com 
To overcome this challenge bone modification is required to accommodate the implant length and size. Bone modification includes, Guided bone regeneration, bone augmentation, Maxillary sinus floor elevation, Alveolar distraction osteogenesis. Modifications in implant design for specific situations but all these modifications are difficult in accessibility, have short-term prognosis and they may result in a prosthetic and functional compromise..$^{(10)}$

We can overcome these challenges by using basal implant basal implantology, also known as bicortical implantology; it can be defined as a modern implantology system which utilizes the basal cortical portion of the jaw bones for retention of the dental implants which are uniquely designed to be accommodated in the basal cortical bone areas. The basal bone provides excellent quality cortical bone for retention of the implants. ${ }^{(11)}$

Basal implantology is a new category of treatment with new broad indications and almost low degree of limitations ${ }^{(12)}$ and considered the first therapy choice in patients with moderately and severely atrophic jaw bone (13) bone build up procedures, nerve displacement, and or sinus lifting doubles the number of surgeries needed and at least doubles the cost of treatment, besides in such protocols $95 \%$ of treatment time is spent on waiting. Basal implants are used to avoid any extra surgical procedures, because these implants anchor reliably in resorption stable cortical bone and they are loaded immediately after surgery.

\section{SUBJECTS AND METHODS}

The present study was conducted on twenty implants for individuals presented with bony defect and seeking for dental implant. They were selected according to inclusion and exclusion criteria.

Both medical and dental histories were taken and investigated, evaluation of the interocclusal space for the placement of the prosthesis, extra and intra oral examination were carried out, hard and soft tissues were evaluated. Patients were assessed using digitalized panoramic radiograph and cone beam computed tomography (CBCT),(Planmeca, HDX Dental OPG, CEPH, 3D Volume Portable, Stationary Anode ).

Determine the size, walls, extension of the bone defect, evaluate the site of implant placement presence or absence of Odontomas or impacted teeth. . Inspect any pathological condition. Evaluate the alveolar bone highest. The mesiodistal and buccolingual dimensions.

Primary impressions were taken and diagnostic study models were casted to evaluate jaw relationships and inter-occlusal space. Surgical stent was fabricated with aid of CBCT to act as a guide for simple and accurate orientation and angulation during implant placement Surgical procedures were performed under local anesthesia (articaine 4\% articaine HCL $68 \mathrm{mg} 1.7 \mathrm{ml}$, Epinephrine $0.017 \mathrm{mg} 1.7 \mathrm{ml}$, art pharmadent for pharmaceutical industries) with buccal and palatal infiltrations. The surgical guide was seated in the patient mouth at its position to avoid injury to vital structures. The flapless approach had been performed to insert BCS implant.

The implant placement procedures were done using the surgical kit of the basal implant (Instrument tray). The drilling had been conducted using adequate cooling with saline to prevent thermal injury to alveolar bone.

\section{Initial drill}

Pike-shaped drill used for easy initiating drilling to prepare a hole of $1.5 \mathrm{~mm}$ diameter and $8 \mathrm{~mm}$ depth, preventing undesired pilot drill sliding at the moment of bone contact.

\section{Pilot drill}

To prepare a hole of $2 \mathrm{~mm}$ diameter and up to $16 \mathrm{~mm}$ depth, which makes it possible to use the forming drill of the first size at the appropriate length. The pilot drill was placed in recipient site in the cortical bone followed by the form drill to widen the recipient site, this so as to allow the thread of the basal implant to successfully engage into this hard 
bone. Cortical bone is very hard and the implant head was not be able to engage it unless a recipient preparation is created.

\section{Form drill}

By the form drill a hole was prepared corresponding to configuration of the implant after applying a pilot drill of the appropriate length.

\section{Implant insertion}

Single stage, single unit BCS basal implant utilized in this study (Dr. Ihde, dental AG (Switzerland) with a suitable length and diameter. Holding key was used to control and maintain implant direction during insertion procedures, insertion tool for external platform (TW 50 torque wrench or a handpiece with a force of up to $40 \mathrm{Ncm}$ ) applied for implant insertion after initial positioning with combined carrier. The BCS implant was immediately placed after drilling. A hand grip, with a considerable amount of axial pressure used to reach the second cortical bone.

\section{Post-operative instructions:}

Medication was prescribed to the patient: Hibiotic $1 \mathrm{gm}$ (500 mg amoxicillin+ 500mg clavulinic acid (AMOUN Pharmaceutic Co) twice daily for 5 days after surgery. Cataflam 50mg (Diclofenac sodium 50mg, Novarts- Switzeland) twice daily for 5 days after surgery.

Oral hygiene instructions including rinsing with Hexitol mouth wash (The arab company for pharmaceutical \& chemical industries ) mouthwash from the second day, for the next 5 days, and a soft diet was recommended.

Impression was taken with rubber base impression material. Implant stability was measured with periotest (Medizintechnik Gulden EK Germany), then a temporary restoration cementation was done. This temporary restoration was removed after six months to allow stability evaluation by the periotest. The twenty patients ranged in age between 48-59 years with a mean age of $55.10 \pm 3.96$ years. Patients were 12 males and 8 females.

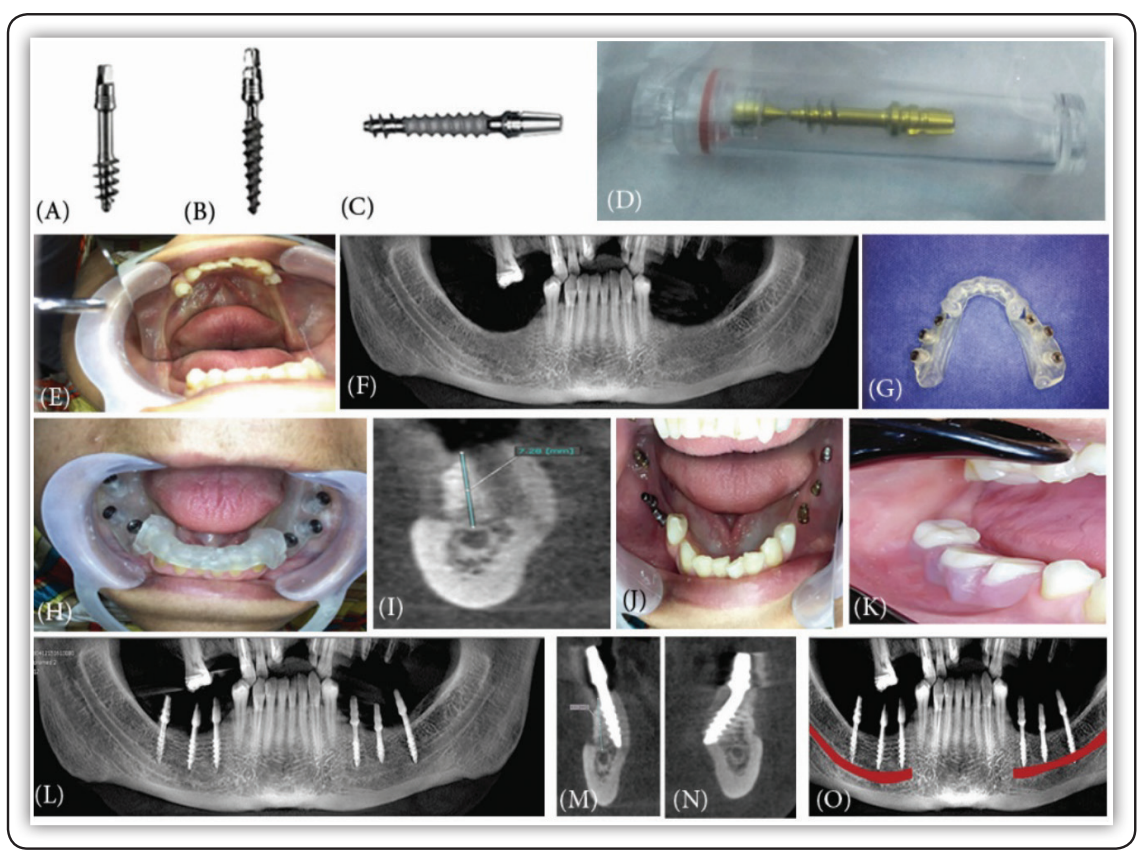

Fig (1)

(a) BCS basal implant (b) KOS basal implant (c) KOS plus basal implant (d) image of BCS basal implant (e) site of implant insertion (f) panoramic radiograph $(\mathrm{g})$ surgical stent (h) surgical guid suited in its place (i) CT showing bone height (j) just after implant insertion (k) temporary restoration () panoramic radiograph showing implantation (m) CT showing implant after insertion (n) CT showing angulation and bending of the implant (o) panoramic radiograph showing six months later on. 


\section{RESULTS}

\section{Stability: Descriptive statistics}

Descriptive statistics of Periotest in the present study are presented in Table (1). The mean Periotest was $0.33 \pm 1.76$ at base line, and $-1.73 \pm 1.71$ after 6 months, figure (1).

TABLE (1) Descriptive statistics of Periotest in each studied period

\begin{tabular}{|c|c|c|c|c|c|c|c|}
\hline Time & Min. & Max. & Mean & \pm SD & Median & \multicolumn{2}{|c|}{$95 \%$ CI } \\
\hline Baseline & -3.0 & 3.0 & 0.33 & 1.76 & 0.0 & -0.64 & 1.31 \\
\hline $\begin{array}{c}\mathbf{6} \\
\text { Months }\end{array}$ & -4.0 & 2.0 & -1.73 & 1.71 & -2.0 & -2.68 & -0.79 \\
\hline
\end{tabular}

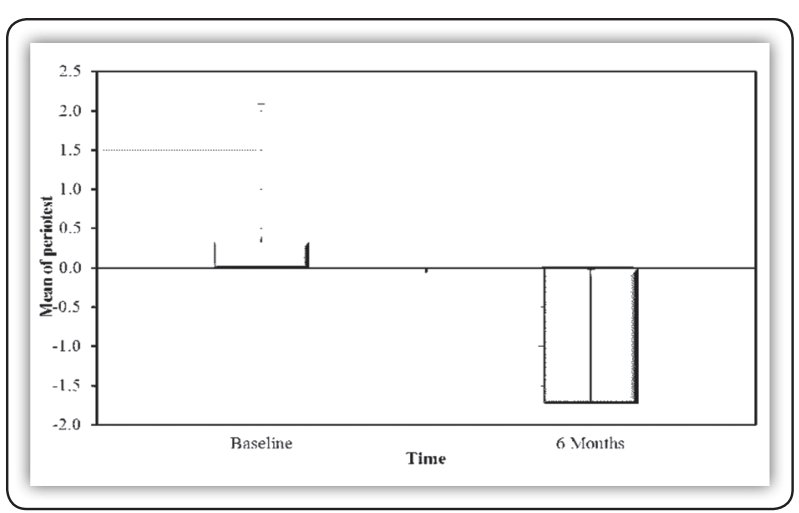

FIG (1) Column chart showing descriptive statistics of Periotest in each period

\section{Changes after treatment}

There was a statistically significant change in mean Periotest $\left(\mathrm{p}=0.002^{*}\right)$ through all periods, table (2). Periotest at 6 months was higher than at baseline with \% of change $\uparrow 36.11 \pm 181.16$, Figure (2).

TABLE (2) Comparison between the two periods according to Periotest

\begin{tabular}{|c|c|c|c|c|c|c|}
\hline & \multicolumn{2}{|c|}{$\begin{array}{c}\text { Baseline } \\
(\mathbf{n}=\mathbf{1 5})\end{array}$} & \multicolumn{2}{c|}{$\begin{array}{c}\text { 6 Months } \\
(\mathbf{n}=\mathbf{1 5})\end{array}$} & \multirow{2}{*}{$\mathrm{Z}$} & \multirow{2}{*}{$\mathbf{p}$} \\
\cline { 1 - 5 } & Mean & \pm SD & Mean & $\mathbf{\pm S D}$ & & \\
\hline Periotest & 0.33 & 1.76 & -1.73 & 1.71 & $3.095^{*}$ & $0.002^{*}$ \\
\hline \% of change & \multicolumn{4}{|c}{$36.11 \pm 181.16$} & & \\
\hline
\end{tabular}

Z: Wilcoxon signed ranks test

$p: p$ value for comparing between Baseline and 6 Months: Statistically significant at $p \leq 0.0$

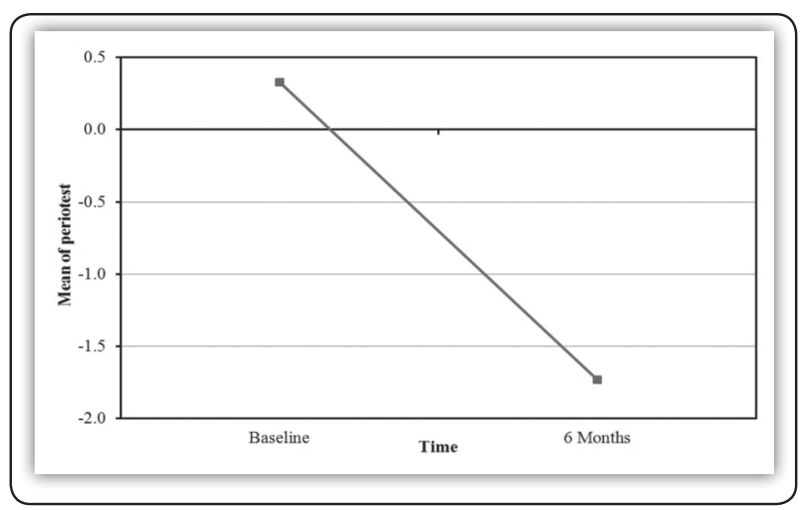

FIG (2) Line chart showing comparison between the two periods according to Periotest

\section{Probing depth (PD)}

\section{Descriptive statistics}

Descriptive statistics of PD in the present study are presented in Table (3). The mean PD was 0.10 $\pm 0.21 \mathrm{~mm}$ at baseline, and $1.52 \pm 0.84 \mathrm{~mm}$ after 6 months, figure (3).

TABLE (3) Descriptive statistics of PD in each studied groups

\begin{tabular}{|c|c|c|c|c|c|c|c|}
\hline Time & Min. & Max. & Mean & \pm SD & Median & \multicolumn{2}{|c|}{$95 \%$ CI } \\
\hline Baseline & 0.0 & 0.50 & 0.10 & 0.21 & 0.0 & -0.05 & 0.25 \\
\hline $\begin{array}{c}\mathbf{6} \\
\text { Months }\end{array}$ & 0.10 & 2.50 & 1.52 & 0.84 & 1.50 & 0.92 & 2.12 \\
\hline
\end{tabular}

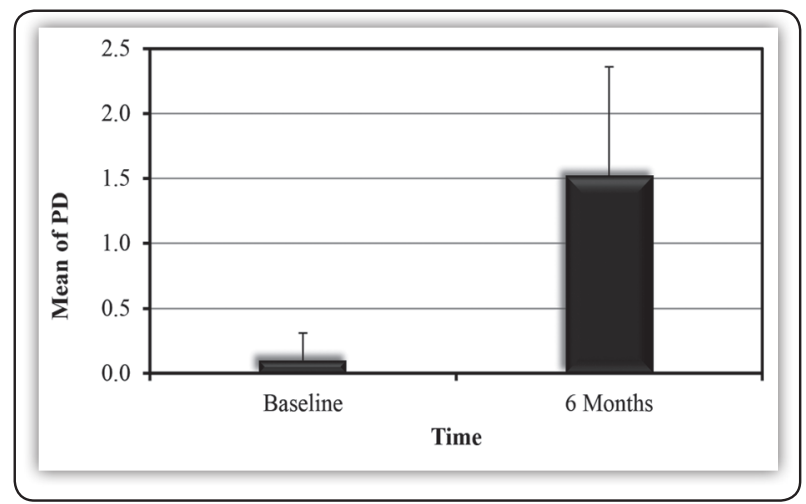

FIG (3) Column chart showing descriptive statistics of PD in each studied groups 


\section{Changes after treatment}

There was a statistically significant change in mean PD through all periods, Table (4), PD at 6 months was higher than at baseline, figure (4).

TABLE (4) Comparison between the two periods according to PD

\begin{tabular}{|c|c|c|c|c|c|c|}
\hline & \multicolumn{2}{|c|}{ Baseline } & \multicolumn{2}{|c|}{6 Months } & \multirow{2}{*}{$\mathbf{Z}$} & \multirow{2}{*}{ p } \\
\hline & Mean & \pm SD & Mean & \pm SD & & \\
\hline PD & 0.10 & 0.21 & 1.52 & 0.84 & $2.812^{*}$ & $0.005^{*}$ \\
\hline$\%$ of change & \multicolumn{4}{|c|}{$200.0 \pm 0.0$} & & \\
\hline
\end{tabular}

Z: Wilcoxon signed ranks test

$p: p$ value for comparing between the studied periods Statistically significant at $p \leq 0.05$

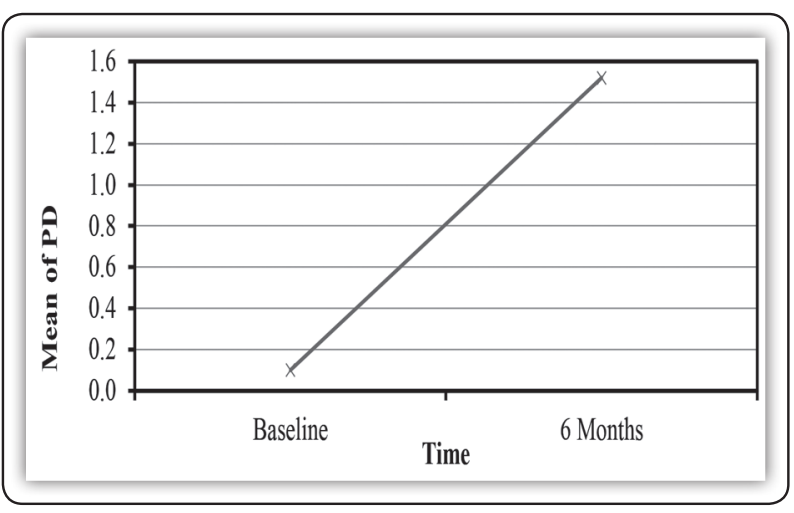

FIG (4) Bar chart showing comparison between the two periods according to $\mathrm{PD}$

\section{Marginal bone loss}

\section{Descriptive statistics}

Descriptive statistics of marginal bone loss in the present study are presented in table (5). The mean marginal bone losswas $0.0 \pm 0.0$ at base line, and $0.60 \pm 0.15$ after 6 months, Figure (5).

TABLE (5) Descriptive statistics of marginal bone loss in each studied groups

\begin{tabular}{|c|c|c|c|c|c|c|c|}
\hline Time & Min. & Max. & Mean & \pm SD & Median & \multicolumn{2}{|c|}{$\mathbf{9 5 \%}$ CI } \\
\hline Baseline & 0.0 & 0.0 & 0.0 & 0.0 & 0.0 & 0.0 & 0.0 \\
\hline 6 Months & 0.40 & 0.80 & 0.60 & 0.15 & 0.60 & 0.49 & 0.71 \\
\hline
\end{tabular}

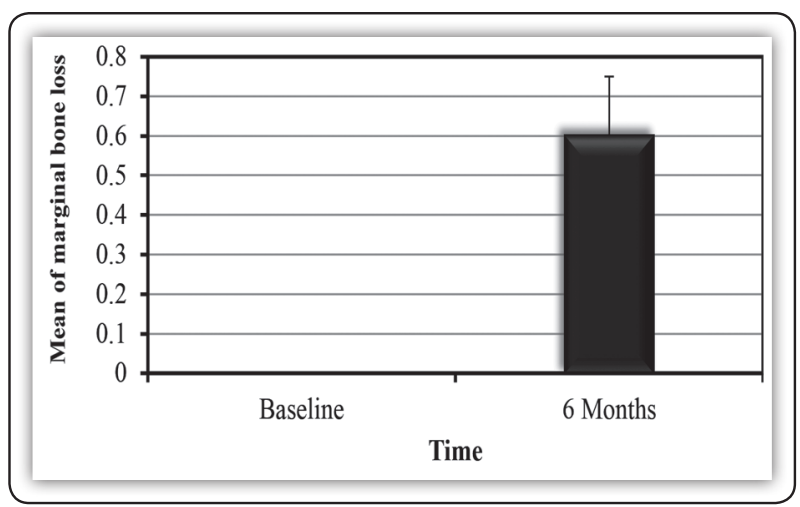

FIG (5) Column chart showing descriptive statistics of marginal bone loss in each period

\section{Changes after treatment}

There was a statistically significant change in mean marginal bone loss through all periods table (6), Marginal bone loss at 6 months was higher than at baseline, Figure (6).

TABLE (6): Comparison between the two periods according to marginal bone loss

\begin{tabular}{|c|c|c|c|c|c|c|}
\hline & \multicolumn{2}{|c|}{ Baseline } & \multicolumn{2}{c|}{ 6 Months } & \multirow{2}{*}{ t } & \multirow{2}{*}{ P } \\
\cline { 2 - 5 } & Mean & $\mathbf{\pm S D}$ & Mean & $\mathbf{\pm S D}$ & & \\
\hline $\begin{array}{c}\text { Marginal } \\
\text { bone loss }\end{array}$ & 0.0 & 0.0 & 0.60 & 0.15 & $12.728^{*}$ & $<0.001^{*}$ \\
\hline $\begin{array}{c}\text { \% of } \\
\text { change }\end{array}$ & \multicolumn{3}{|c}{-} & & & \\
\hline
\end{tabular}

$t$ : Paired t-test

$p: p$ value for comparing between the studied periods

*: Statistically significant at $p \leq 0.05$

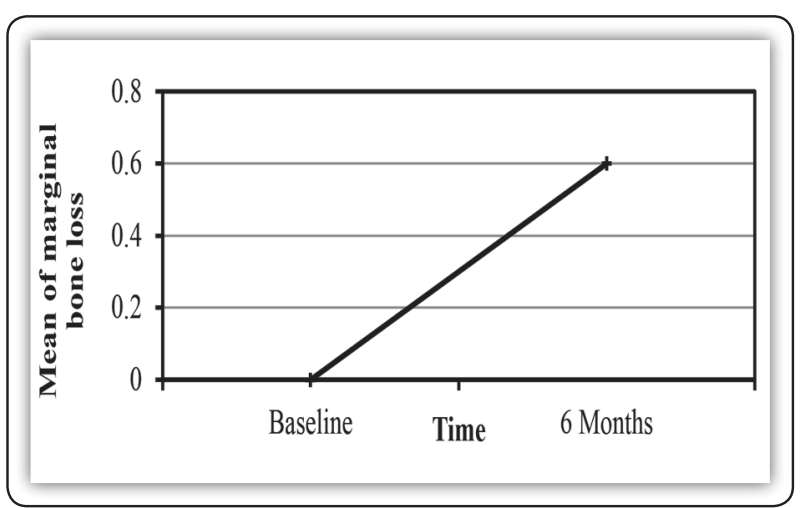

FIG (6) Line chart showing comparison between the two periods according to marginal bone loss. 


\section{DISCUSSION}

For any successful implant placement it is predicted the bone height should be minimum 13$15 \mathrm{~mm}$ and the width should be minimum 5-7 mm. When the available bone is not sufficient, procedures such as bone grafts, GBR, bony expansion using osteotomes, ridge splitting technique, alveolar distraction, maxillary sinus floor elevation, nerve repositioning and basal implantology can be used ${ }^{(14)}$.

Procedures described above to augment bone and deal with anatomical structures have their own challenges. Bone grafting, osseodistraction and sinus lifts are invasive procedures. In addition, they add complexity and increase the number of surgical phases required for implant therapy with Bone grafting, whether autogenous or allogenous, carries with it a risk of complications that include the harvesting procedure itself (for autogenous grafts) and the possibility of graft infection, poor flap closure, dehiscence and resorption of the graft.

Basal implantology also known as bicortical implantology or cortical implantology is a modern implantology system which utilizes the basal cortical portion of the jaw bones for retention of the dental implants which are uniquely designed to be accommodated in the basal cortical bone areas. The basal bone provides excellent quality cortical bone for retention of these unique and highly advanced implants. The two types basal osseointegrated and basal cortical screw (BCS) basal implants are specifically designed to utilize strong cortical bone of the jaw. Screwable basal implants (BCS brand) have been developed with up to $12 \mathrm{~mm}$ thread diameter can be inserted into immediate extraction socket ${ }^{(15)}$.

For the past two decades cases with resorbed ridges and narrow ridges are treated with basal implant system. The basal implant system is most successful in such cases also with minimally invasive technique. Basal implants with compression types serves as a better alternative in such resorbed ridges.
Basal implants are mainly indicated in cases with resorbed ridge and where the bone height from the limiting structure is compromised (16).

Immediate loading of basal implants can be done, when they are placed in the dense cortical bone, as they attain high primary stability there. Therefore, they are more predictable than before, though there are high chances of crestal bone loss. Since the remodeling of the bone starts within $72 \mathrm{~h}$ and weakens the peri-implant bone structures, rigid splinting of the metal framework should be done as early as possible. About $100 \%$ success rate can be achieved if BCS implants are used along with an appropriate immediate load protocol ${ }^{(17)}$.

The high stability of basal implant can be attributed to that the basal implant is inserted in two places of cortical bone. The cortical bone used for anchoring the basal implant is highly mineralized, and is not absorbed through the course of life. BCS (IHDE) basal implant that used in this study is a single piece with a polished sharp apical threads for placement in the cortical bone ${ }^{(18)}$.

Regarding the implant probing depth and considering the marginal bone loss, there were highly satisfaying records. Reduced bone loss in basal implants may be due to that it is polished at the neck to prevent bacterial colonization thus decreasing incidence of peri-implantitis and then bone resorption.

Recently in 2015 Mahender Singh et al ${ }^{(15)}$ did a study on a novel approach for restoration of hemisected mandibular first molar with immediately loaded single piece BCS implant and replacement of the mesial root with a single piece immediate loaded BCS (Bi-Cortical Screw) implant. He found that there was no bone loss around the BCS.

Basal implants are mainly indicated in cases with resorbed ridge and where the bone height from the limiting structure is compromised (16). Basal implants are one piece implants that minimizes the failure of implants due to the interface problems 
between the connections that exist in conventional two and three piece implants.

These implants take support from the basal bone which is a lot more resistant to resorption, unlike the conventional implants that mostly take support from the Crestal bone. Basal cortical bone has a much faster and stable repairing capacity. Bone augmentation / grafting, sinus lifting and nerve transpositioning procedures can be avoided. These implants particularly take advantage of the basal bone available to avoid bone augmentation procedures.

Whereas for conventional implants the available bone has to be modified/augmented by surgical procedures to accept the implants. The basal implants are imbedded in high quality basal bone. Hence, the masticatory forces get distributed to the cortical bone areas that are highly resistant to resorption and have a very high repairing capacity ${ }^{(15)}$. In the present study, there was a statistically significant change in mean marginal bone loss through all periods. Marginal bone loss at 6 months was higher than at baseline.

Peri-implantitis is the common etiology leading to failure of conventional implants. This occurs mostly due to the roughness of the implant surface along with the interface problems between the multiple parts of the implant. The monobloc smooth surface basal implants eliminates the threat of periimplantitis in around $98 \%$ of cases ${ }^{(15)}$. There was a statistically significant change in mean PD through all periods. PD at 6 months was higher than at baseline.

Masticatory forces transmitted through the basal implants may create local microcracks in the cortical bone. These microcracks are repaired by formation of secondary osteotomes, a process called as remodelling. However, this temporarily reduces the degree of mineralization and increases the porosity of the affected bone. Hence, basal implants have a good chance of reintegration, if loads are reduced to an adequate amount.
In accordance, in the present study, there was a statistically significant change in mean Periotest through all periods. Periotest records at 6 months were lesser than at baseline.

Evolution of basal implants have given positive hope for the patients with atrophied ridges which can be rehabilitated not only by avoiding augmentation procedures, time, and cost but also by immediately loading of the prosthesis making them more confident and socialize normally.

It can be concluded that, basal dental implant is an excellent choice in resorbed and narrow ridge when compare to other invasive procedures. The implant success rate is also high when compare to the other treatment protocol. This implant system also has an advantage of immediate loading which was impossible in other systems.

\section{CONCLUSION}

Basal implant can be used successfully in severely atrophied ridge and compromised bone. Basal implants are used to avoid any extra surgical procedures, because these implants anchor reliably in resorption stable cortical bone and they are loaded immediately after surgery.

\section{REFERENCES}

1. Tallgren A. The continuing reduction of the residual alveolar ridges in complete denture wearers: a mixed-longitudinal study covering 25 years. The Journal of Prosthetic Dentistry. 2003;89(5):427-3

2. Pinho MrN, Roriz VlM, Novaes AB, Taba Mr, Grisi MrFM, de Souza SrLS, et al. Titanium Membranes in Prevention of Alveolar Collapse After Tooth Extraction . Implant Dentistry. 2006;15(1):53-61

3. Lekovic V, Camargo PM, Klokkevold PR, Weinlaender M, Kenney EB, Dimitrijevic B, et al. Preservation of Alveolar Bone in Extraction Sockets Using Bioabsorbable Membranes. Journal of Periodontology. 1998;69(9):1044-9

4. Schropp L, Wenzel A, Kostopoulos L, Karring T. Bone healing and soft tissue contour changes following singletooth extraction: a clinical and radiographic 12-month prospective study. International Journal of Periodontics Restorative Dentistry. 2003;23 
5. Wang K, Li DH, Guo JF, Liu BL, Shi SQ. Effects of buccal bicortical anchorages on primary stability of dental implants: a numerical approach of natural frequency analysis. Journal of Oral Rehabilitation. 2009;36(4):284-91

6. Ding X, Zhu X-H, Liao S-H, Zhang X-H, Chen H. Implant-Bone Interface Stress Distribution in Immediately Loaded Implants of Different Diameters: A Three-Dimensional Finite Element Analysis. Journal of Prosthodontics. 2009;18(5):393-402.

7. Meredith N, Books K, Fribergs B, Jemt T, Sennerby L. Resonance frequency measurements of implant stability in viva. A crosssectional and longitudinal study of resonance frequency measurements on implants in the edentulous and partially dentate maxilla. Clinical Oral Implants Research. 1997;8(3):226-33.

8. Mohammed Ibrahim M, Thulasingam C, Nasser KSGA, Balaji V, Rajakumar M, Rupkumar P. Evaluation of Design Parameters of Dental Implant Shape, Diameter and Length on Stress Distribution: A Finite Element Analysis. The Journal of Indian Prosthodontic Society. 2011;11(3):165-71

9. Toniollo MB, Macedo AP, Rodrigues RCS, Ribeiro RF, de Mattos MdGC. Three-Dimensional Finite Element Analysis of Stress Distribution on Different Bony Ridges With Different Lengths of Morse Taper Implants and Prosthesis Dimensions. Journal of Craniofacial Surgery. 2012;23(6):1888-92.

10. Agliardi E, Panigatti S, Clericò M, Villa C, Malò P. Immediate rehabilitation of the edentulous jaws with full fixed prostheses supported by four implants: interim results of a single cohort prospective study. Clinical Oral Implants Research. 2010;21(5):459-65

11. Lekovic V, Camargo PM, Klokkevold PR, Weinlaender M, Kenney EB, Dimitrijevic B, et al. Preservation of Alveolar Bone in Extraction Sockets Using Bioabsorbable Membranes. Journal of Periodontology. 1998;69(9):1044-9.

12. Misch C, Dietsh F. Autogenous bone grafts for endosteal implants--indications and failures. The International journal of oral implantology: implantologist. .1991;8(1):13-20

13. Van Steenberghe D, Lekholm U, Bolender C, Folmer T, Henry P, Herrmann I, et al. The Applicability of Osseointegrated Oral Implants in the Rehabilitation of Partial Edentulism: A Prospective Multicenter Study on 558 Fixtures. International Journal of Oral Maxillofacial Implants. 1990;5

14. Gupta AD, Verma A, Dubey T, Thakur S. Basal osseointegrated implants: Classification and review. Int J Contemp Med Res. 2017;4(11):2329-33

15. Yadav R, Sangur R, Mahajan T, Rajanikant A, Singh N, Singh R. An alternative to conventional dental implants: Basal implants. Rama Univ J Dent Sci. 2015;2(2):22-8

16. Nair C, Bharathi S, Jawade R, Jain M. Basal implants-a panacea for atrophic ridges. Journal of dental sciences oral rehabilitation. 2013:1-4

17. Ihde $\mathrm{S}, \mathrm{A}$ I. The International Implant Foundation Immediate Loading. Munich, Germany:2011.

18. Weber H, Fiorellini J. The biology and morphology of the implant tissue interface The Alpha omegan. 1992; 85(4):61-4. 